\title{
Lamotrigine for bipolar I disorder improved depressive symptoms on some depression scales
}

\author{
Calabrese JR, Bowden CL, Sachs GS, et al, for the Lamictal 602 Study Group. A double-blind placebo-controlled study of lamotrigine \\ monotherapy in outpatients with bipolar I depression. J Clin Psychiatry 1999 Feb;60:79-88.
}

\section{Question}

In patients with bipolar I disorder, is lamotrigine effective for treating a major depressive episode?

\section{Design}

7 week randomised, double blind, placebo controlled trial.

\section{Setting}

15 centres in the US and 6 in the UK, France, and Australia.

\section{Patients}

195 patients who were $\geqslant 18$ years of age (mean age $42 \mathrm{y}, 61 \%$ women) with bipolar 1 disorder according to DSM-IV criteria and a current major depressive episode (score $\geqslant 18$ on the 17 item Hamilton Rating Scale for Depression [HAM-D]) lasting $2-52$ weeks. Patients had had $\geqslant 2 \operatorname{mood}$ episodes with $\geqslant 1$ manic or mixed episode in the previous 10 years. Exclusion criteria were rapid cycling bipolar disorder; abnormal thyroid function tests; panic disorder, obsessive compulsive disorder, social phobia, or bulimia nervosa in the previous 12 months; substance abuse or dependence; pregnancy; lactation; suicidal tendency; or serious or unstable medical conditions. $69 \%$ of patients completed the study, and $98 \%$ were analysed.

\section{Intervention}

Patients were allocated to lamotrigine, $50 \mathrm{mg} /$ day $(\mathrm{n}=66)$ or 200 $\mathrm{mg} /$ day $(\mathrm{n}=63)$, or placebo $(\mathrm{n}=66)$ in tablets given twice daily.

\section{Main outcome measures}

Treatment response (defined as $\geqslant 50 \%$ reduction in score on the HAM-D or Montgomery-Asberg Depression Rating Scale [MADRS] or a rating of very much improved or much improved on the Clinical Global Impressions scale for Improvement [CGI-I]).

\begin{abstract}
Main results
Analysis was by intention to treat. Differences in mean HAM-D scores for lamotrigine and placebo were not statistically significant. Lamotrigine, $200 \mathrm{mg} /$ day, led to more improved scores than did placebo on the MADRS (mean score difference $13.3 v 7.8, \mathrm{p}<0.05$ ) and CGI-I (mean score difference 2.6 v 3.3, $\mathrm{p}<0.05)$. Both lamotrigine groups had greater responder rates on the MADRS than did the placebo group $(\mathrm{p}<0.05$ for both comparisons) (table). Lamotrigine, $200 \mathrm{mg} /$ day, led to greater responder rates on the CGI-I than did placebo $(\mathrm{p}<0.05)$ (table).
\end{abstract}

\section{Conclusion}

In patients with bipolar I disorder and a major depressive episode, lamotrigine was effective for improving depressive symptoms on some depression scales.

Responder rates for lamotrigine (Lam) v placebo in bipolar I disorder*

\begin{tabular}{llllll}
\hline Lam dose & Scale & Lam & Placebo & RBI (95\% CI) & NNT (CI) \\
\hline $50 \mathrm{mg}$ /day & HAM-D & $45 \%$ & $37 \%$ & $23 \%(-19$ to 87$)$ & Not significant \\
& MADRS & $48 \%$ & $29 \%$ & $66 \%(6$ to 163$)$ & 6 (3 to 43) \\
& CGI-I & $41 \%$ & $26 \%$ & $55 \%(-5$ to 159$)$ & Not significant \\
$200 \mathrm{mg}$ /day & HAM-D & $51 \%$ & $37 \%$ & $38 \%(-7$ to 107$)$ & Not significant \\
& MADRS & $56 \%$ & $29 \%$ & $90 \%(24$ to 198$)$ & 4 (3 to 11) \\
& CGI-I & $51 \%$ & $26 \%$ & $94 \%(23$ to 215$)$ & 5 (3 to 13) \\
\hline
\end{tabular}

*HAM-D $=17$ item Hamilton Rating Scale for Depression; MADRS $=$ Montgomery-Asberg Depression Rating Scale; CGI-I = Clinical Global Impressions scale for Improvement. Other abbreviations defined in glossary; RBI, NNT, and CI calculated from data supplied by author.

Source of funding: Glaxo Wellcome Research and Development.

For correspondence: $\operatorname{Dr} J$ R Calabrese, Case Western Reserve University, 11400 Euclid Avenue, Suite 200, Cleveland, Ohio 44106, USA.Fax +1 2167950300.

\section{Commentary}

The question posed is clinically important given the poor response of patients with bipolar depression to lithium and the switching to mood elevation or rapid cycling associated with many antidepressant medications. As the first randomised, parallel group, placebo controlled trial of lamotrigine for bipolar depression, it provides the most robust answer to date.

In this study, the clinical significance of the CGI-I score as a measure of treatment response is easier to appreciate than the MADRS and HAM-D. The CGI-I number needed to treat of 5 (95\% CI 3 to 13) for lamotrigine, $200 \mathrm{mg}$ /day for 7 weeks, is similar to that seen in pharmacotherapy for unipolar depressive disorders. ${ }^{12}$

Lamotrigine does not induce P450 isoenzymes, has no substantial pharmacokinetic effects on concentrations of commonly used psychotropic medica- tions, and was well tolerated in this trial. Several cases of delirium have been reported. ${ }^{3}$ Rare events possibly associated with lamotrigine include StevensJohnson syndrome, neutropenia, thrombocytopenia, and agranulocytosis. ${ }^{4}$

The patient characteristics in this study help to inform the application of its results to clinical practice. At baseline, most patients had a "moderately ill" CGI-S score and all were non-suicidal outpatients. Exclusion of patients with substance abuse or dependence and other relatively common conditions will limit further the generalisation of these data to clinical practice. Ultimately, appropriate application of this trial in clinical practice may best be guided by deciding whether the clinical and physiological features of a patient with bipolar I disorder and a current major depressive episode are so unlike those in this study that the results should not apply.

Clinicians await a similarly robust trial comparing lamotrigine with a mood stabiliser or antidepressant for patients with bipolar depression. This would more closely approximate standard practice. Until then, lamotrigine's place in the treatment of these patients remains unclear.

\section{David Haslam, MD, MS Dalhousie University Halifax, Nova Scotia, Canada}

1 Evidence-Based Mental Health 1998;1:49. Abstract of: Emslie G], Rush A], Weinberg WA, et al. Arch Gen Psychiatry 1997;54:1031-

2 Evidence-Based Mental Health 1998;1:111. Abstract of: Lima MS, Moncrieff J. (Cochrane Review, latest version 5 Jan 98). In: Cochrane Library. Oxford: Update Software.

3 Hennessey MJ, Wiles CM. [letter]. Lancet 1996; 347:974-5.

Mackay FJ, Wilton LV, Pearce GL, et al. Epilepsia 1997:38:881-6. 